\title{
Imbalance of prostacyclin and thromboxane synthesis in Crohn's disease
}

\author{
C J HAWKEY, FANNY KARMELI, AND D RACHMILEWITZ \\ From the Nuffield Department of Clinical Medicine, John Radcliffe Hospital, Oxford, and Department of \\ Gastroenterology, Hadassah University Hospital, Jerusalem, Israel
}

SUmmary Synthesis of prostanoids in Crohn's disease was investigated using rectal biopsy specimens maintained in organ culture. As with ulcerative colitis increased synthesis of prostaglandin $(\mathrm{PG}) \mathrm{E}_{2}$ was observed when the mucosa was inflamed, compared with uninflamed mucosa in Crohn's disease, and with control biopsy specimens. In contrast with ulcerative colitis differences from control specimens were observed even in the absence of inflammation. There was a raised synthesis of thromboxane $(\mathrm{Tx}) \mathrm{B}_{2}$ (stable breakdown product of $\mathrm{TxA}_{2}$ ); concentrations of 6-keto $\mathrm{PGF}_{1 \alpha}$ (stable breakdown product of prostacyclin) were unchanged and hence the ratio of 6-keto $\mathrm{PGF}_{1 \alpha} / \mathrm{TxB}_{2}$ was reduced. These changes might lead to an altered cytoprotective capacity or reduced suppressor cell activity, such as has previously been reported in intestinal lymphocytes in Crohn's disease.

In ulcerative colitis several studies have shown that increased synthesis of prostanoids such as $\mathrm{PGE}_{2}$, $\mathrm{PGF}_{2 \alpha}, \mathrm{TxB}_{2}$, and 6-keto $\mathrm{PGF}_{1 \alpha}$ occurs during relapse. $^{1-4}$ Fundamental differences from normal have not been shown in remission. The significance of these findings is unclear as thromboxane $A_{2}$ and prostacyclin have powerful opposing actions and it has been suggested that an imbalance in the synthesis of these two prostanoids may be of pathological significance in several disease states. ${ }^{5}$

Data on mucosal prostaglandin synthesis in Crohn's disease are lacking although peripheral blood mononuclear cells have been shown to synthesise increased amounts of $\mathrm{PGE}_{2}$ and $\mathrm{TxB}_{2}$ during relapse. ${ }^{6}$ In the present study we have investigated basal synthesis of $\mathrm{PGE}_{2}, \mathrm{TxB}_{2}$, and 6-keto $\mathrm{PGF}_{1 \alpha}$ by rectal mucosa from patients with Crohn's disease maintained in organ culture. The study shows a deviation from normal in the synthesis of $\mathrm{TxB}_{2}$ and its relationship to 6-keto $\mathrm{PGF}_{1 \alpha}$ even in the absence of inflammation.

\section{Methods}

PATIENTS

Seventeen patients with Crohn's disease (mean age 40.7 $\pm 3 \cdot 6 \mathrm{SEM}$ years) were studied and compared

Address for correspondence: Dr C J Hawkey, Department of Therapeutics, University Hospital, Nottingham NG7 2UH.

Received for publication 29 December 1982 with seven control patients (mean age $53 \cdot 1 \pm 9 \cdot 3$ years). All the patients studied were British. The diagnosis of Crohn's disease was established on the basis of histology of biopsy or resected specimens and on radiological appearances. Details of the distribution of the Crohn's disease based upon evidence available at the time of biopsy are shown in the Table.

Inflammation was judged on sigmoidoscopic appearances supported by a routine histological examination. All biopsies that were considered inflamed came from reddened areas with loss of vascular pattern, granularity, and either spontaneous haemorrhage or haemorrhagic friability. The mucosa was considered uninflamed if it was pearly with a clearly visible vascular pattern and no sign of haemorrhage. Biopsies from equivocal

Table Details of patients studied

\begin{tabular}{lll}
\hline & $\begin{array}{l}\text { Crohn's disease } \\
(10 \text { men, 7 women })\end{array}$ & $\begin{array}{l}\text { Control } \\
(5 \text { men, } \\
2 \text { women })\end{array}$ \\
\hline Age & $40 \cdot 7 \pm 3 \cdot 6$ & $53 \cdot 1 \pm 9 \cdot 3$ \\
Distribution & & \\
$\quad$ Small bowel/caecal & 4 & \\
$\quad$ Small + large bowel & 5 & \\
Large bowel & 8 & \\
\hline
\end{tabular}

All the patients with large bowel Crohn's disease had rectal involvement, though some were uninflamed at the time of biopsy. 
mucosa were not used. At the time of study the rectal mucosa was diffusely inflamed in seven cases; five patients had rectal disease sufficiently localised for biopsy specimens from both sigmoidoscopically inflamed and uninflamed mucosa to be taken. In six cases the rectal mucosa was uninflamed and in four of these the disease was apparently confined to the small bowel or ileocaecal region and there had never been evidence of rectal involvement.

The drugs used in the treatment of Crohn's disease at the time of biopsy were sulphasalazine $(n=4)$, prednisolone $(n=1)$, sulphasalazine and prednisolone $(n=3)$, or sulphasalazine and azathioprine $(n=2)$; seven patients were untreated at the time of biopsy. The control biopsy specimens were taken during the investigation of untreated patients with irritable colon syndrome $(n=2)$, gastrointestinal blood loss $(n=2)$, transient diarrhoea $(n=1)$, small bowel lymphoma $(n=1)$, and for the exclusion of amyloid $(n=1)$. The specimens were histologically normal and in none was any organic disease involving the rectum shown.

Rectal biopsy specimens were excised 6 to $10 \mathrm{~cm}$ from the anus by forceps biopsy. The median biopsy weights were $8 \mathrm{mg}$ (range 5-18 $\mathrm{mg}$ ) for the patients with Crohn's disease and $13 \mathrm{mg} \mathrm{(3-16} \mathrm{mg)} \mathrm{for} \mathrm{the}$ control patients. After excision the biopsy specimens were washed in RPMI 1640 culture medium and cleaned of blood. They were placed in organ culture ${ }^{23}$ using RPMI 1640 containing penicillin $(100 \mathrm{U} / \mathrm{ml})$ and streptomycin $(100 \mu \mathrm{g} / \mathrm{ml})$ and cultured at $37^{\circ} \mathrm{C}$ in a vacuum jar containing $95 \%$ oxygen $/ 5 \% \mathrm{CO}_{2}$. No fetal calf serum was added to the medium as albumin interferes with the assay of $\mathrm{TxB}_{2} .{ }^{4}$ The culture medium was changed after one hour and the culture continued for a further 23 hours. The synthesis of $\mathrm{PGE}_{2}, \mathrm{TxB}_{2}$, and 6-keto $\mathrm{PGF}_{1 \alpha}$, judged by their accumulation in the medium at the end of culture, was measured, as previously described, ${ }^{4}$ by specific radioimmunoassays, performed without knowledge of the source of the samples.

$$
\text { ○ Rectum uninvolved } \quad \bullet \text { Rectum }
$$
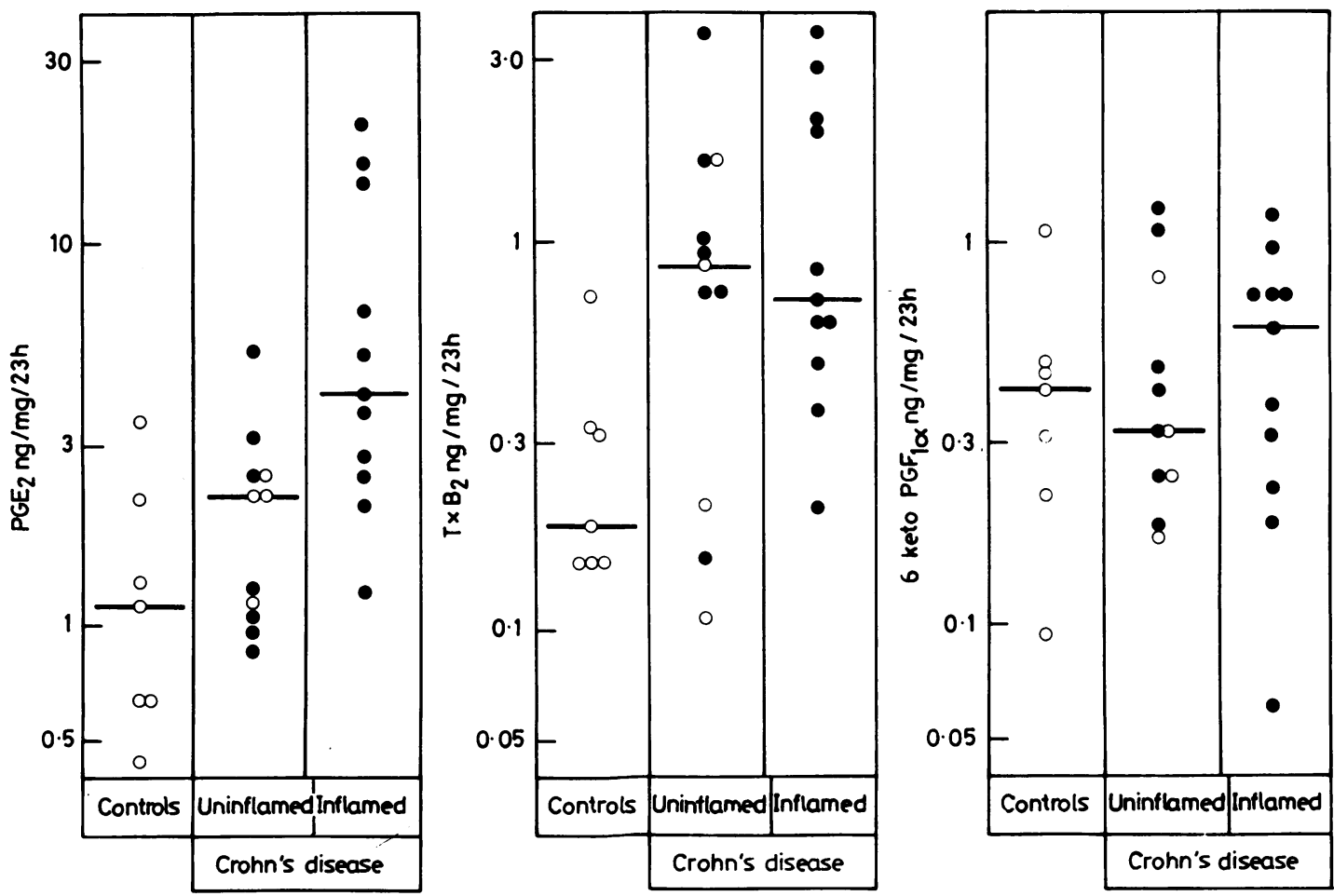

Fig. 1 Synthesis of prostanoids in organ culture. Data from individual biopsy specimens are shown. For each prostanoid synthesis is expressed as $\mathrm{ng} / \mathrm{mg}$ wet weight over 23 hours. 


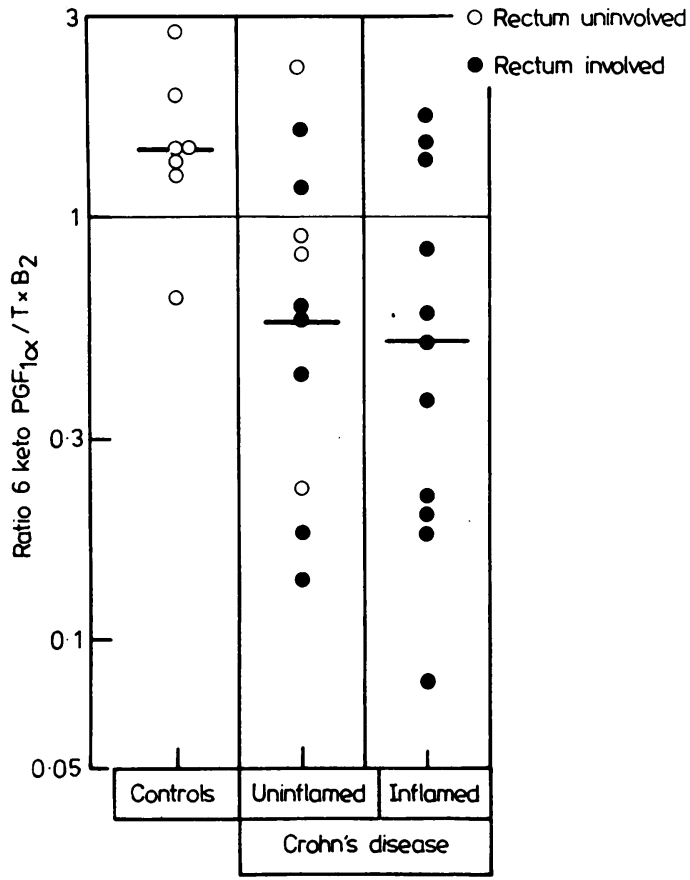

Fig. 2 Ratio of 6-keto $P G F_{1 \alpha} / T x B_{2}$ synthesis. Data from individual biopsy specimens are shown. For each specimen the synthesis of 6-keto $P G F_{l \alpha}$ (in $n g / m g$ wet weight/23 hours) has been expressed as a proportion of the synthesis of $T x B_{2}$ (in $n g / m g$ net weight $/ 23$ hours).

\section{STATISTICAL METHODS}

As with prostaglandin synthesis in ulcerative colitis the distribution of the results was skewed and comparisons between groups were, therefore, made by the non-parametric Mann Whitney $U$ test, using two tailed significance values. In the text median values are quoted and the range of individual values is shown in Figs 1 and 2. Paired comparisons between inflamed and uninflamed mucosa from patients with local rectal involvement were evaluated using the Sign test.

\section{Results}

(i) $P G E_{2}$ : Inflamed mucosa from patients with Crohn's disease $(n=12)$ synthesised significantly more $\mathrm{PGE}_{2}$ than uninflamed mucosa $(n=11)$ from patients with Crohn's disease (median values 4.67 $\mathrm{ng} / \mathrm{mg}, 13.34 \mathrm{pmol} / \mathrm{mg}$ wet weight vs $2.22 \mathrm{ng} / \mathrm{mg}$, $6.34 \mathrm{pmol} / \mathrm{mg}, \mathrm{p}<0.02)$. The synthesis of $\mathrm{PGE}_{2}$ by the seven control biopsies (median $1.25 \mathrm{ng} / \mathrm{mg}, 3.57$ $\mathrm{pmol} / \mathrm{mg}$ ) did not differ significantly from that seen with uninflamed mucosa in Crohn's disease $(p>0 \cdot 10)$ but was significantly lower than the amount of $\mathrm{PGE}_{2}$ synthesised by the inflamed mucosa $(p=0 \cdot 02)$. The range of individual values is shown in Fig. 1.

Where paired comparisons were made in patients with patchy involvement of the rectum all five showed greater synthesis of $\mathrm{PGE}_{2}$ by sigmoidoscopically inflamed than by uninflamed mucosa.

(ii) $T \times B_{2}$ : The median amounts of $\mathrm{TxB}_{2}$ synthesised in Crohn's disease were $0.70 \mathrm{ng} / \mathrm{mg}, 1.89 \mathrm{pmol} / \mathrm{mg}$ (inflamed mucosa $\mathrm{n}=11$ ) and $0.90 \mathrm{ng} / \mathrm{mg}, 2.43$ $\mathrm{pmol} / \mathrm{mg}$ (uninflamed mucosa $\mathrm{n}=11$ ). These values did not differ significantly from each other but with both inflamed and uninflamed mucosa the synthesis of $\mathrm{TxB}_{2}$ was significantly higher than was observed in the control patients (median synthesis $0.18 \mathrm{ng} / \mathrm{mg}$, $0.49 \mathrm{pmol} / \mathrm{mg}, \mathrm{n}=7, \mathrm{p}<0.05$ compared with uninflamed, $\mathrm{p}<0.02$ compared with inflamed mucosa from patients with Crohn's disease). High rates of $\mathrm{TxB}_{2}$ synthesis were seen in two of the patients with Crohn's disease where the rectum was apparently uninvolved.

(iii) 6-Keto $P G F_{1 \alpha}$ : No significant differences could be shown for the synthesis of 6-keto PGF $_{1 \alpha}$ between inflamed (median $0.63 \mathrm{ng} / \mathrm{mg}, 1.70 \mathrm{pmol} / \mathrm{mg}$ ) or uninflamed mucosa (median $0.33 \mathrm{ng} / \mathrm{mg}, 0.89 \mathrm{pmol} /$ $\mathrm{mg}$ ) from patients with Crohn's disease or uninflamed mucosa from the control patients (median $0.41 \mathrm{ng} / \mathrm{mg}, 1.11 \mathrm{pmol} / \mathrm{mg}$ ). The ratio of 6-keto $\mathrm{PGF}_{1 \alpha}$ to $\mathrm{TxB}_{2}$ synthesis, however, was significantly higher in the control biopsy specimens (median ratio 1.47) compared with both inflamed (median ratio $0.52 ; \mathrm{p}<0.05$ ) and uninflamed mucosa (median ratio $0.62, \mathrm{p}<0.02$ ) from patients with Crohn's disease. The difference between the control biopsy specimens and those with uninflamed mucosa with Crohn's disease remains significant $(p=0.05)$ if the uninflamed biopsy specimens from patients with localised inflammation are excluded.

Six of the seven control patients showed a ratio greater than 1.0 whereas in only six out of 22 of the specimens from patients with Crohn's disease was it greater than 1.0. These results are shown in full in Fig. 2.

\section{Discussion}

As with previous studies concerning ulcerative colitis $^{1-4}$ the synthesis of $\mathrm{PGE}_{2}$ by inflamed rectal mucosa has been shown to be enhanced in Crohn's disease. This may be a secondary consequence of invasion of the lamina propria by inflammatory mononuclear cells, but it may nevertheless contribute to the modulation of local inflammatory and immune responses. The finding of increased $\mathrm{TxB}_{2}$ synthesis by rectal mucosa from patients with 
Crohn's disease, even in the absence of inflammation, was unexpected but might be of pathogenic significance. It could have arisen artefactually as a result of occult inflammation in apparently normal mucosa but against this proposition is the fact that an analogous effect was not shown for $\mathrm{PGE}_{2}$. Furthermore, the raised synthesis of $\mathrm{TxB}_{2}$ in two patients who had never had apparent rectal involvement raises the possibility - which requires confirmation - that this change may occur in the absence of clinically evident disease. As with ulcerative colitis ${ }^{2-4}$ there is a wide scatter of results and overlap between individuals of different groups. The findings of a significant rise of thromboxane synthesis and a significant reduction in the 6-keto $\mathrm{PGF}_{1 \alpha} / \mathrm{TxB}_{2}$ ratio in Crohn's disease even in the absence of inflammation thus apply to the groups rather than to all individuals.

These differences between the groups could have arisen in two ways. The most obvious explanation is that they arose because of a fundamental difference between the control patients and the patients with Crohn's disease. Most of the patients with Crohn's disease, however, were taking sulphasalazine, prednisolone, or azathioprine and an alternative explanation is that the results arose as a result of a pharmacological effect of treatment. If this were so our results could be in contradiction to data from previous work. This has shown that sulphasalazine, 5-amino salicylic acid, corticosteroids, and azathioprine can inhibit thromboxane and prostaglandin synthesis by rectal mucosa,,$^{2-4} 7$ that under some circumstances 5-amino salicylic acid can enhance synthesis of prostacyclin and $\mathrm{PGF}_{2 \alpha},{ }^{8}$ and that sulphasalazine can inhibit degradation of $\mathrm{PGE}_{2}$ and $\mathrm{PGF}_{2 \alpha}{ }^{9}{ }^{9}$ On the basis of these data one would predict that treatment with sulphasalazine, corticosteroids, and azathioprine would lead to reduced rather than enhanced synthesis of $\mathrm{TxB}_{2}$; if the increased synthesis of $\mathrm{TxB}_{2}$ and reduced 6-keto $\mathrm{PGF}_{1 \alpha} / \mathrm{TxB}_{2}$ ratio we have observed in Crohn's disease is due to the disease process, treatment with sulphasalazine could oppose these changes both by reducing thromboxane synthesis and by enhancing prostacyclin synthesis. ${ }^{48}$

Increased thromboxane synthesis may occur as a result of platelet activation but the results are unlikely to be due to contamination by blood as the biopsies were meticulously cleaned of blood and rejected if contaminated. Cells other than platelets are more likely to be the source of thromboxane for example, macrophages or other leucocytes. ${ }^{10}$ Synthesis of prostanoids by peripheral blood mononuclear cells has been shown to be elevated in Crohn's disease, ${ }^{5} \mathrm{TxB}_{2}$ appears to be the predominant product of the cyclo-oxygenase pathway of human monocytes and neutrophils; these cells synthesise little if any prostacyclin ${ }^{10}$ and their accumulation in the mucosa may account for the rise of $\mathrm{TxB}_{2}$ synthesis and the altered 6-keto $\mathrm{PGF}_{1 \alpha} / \mathrm{TxB}_{2}$ ratio that we have shown in Crohn's disease. The inflammatory mediators leukotrienes C4 and D4 are potent stimuli for the synthesis of $\mathrm{TxB}_{2}$ by rat peritoneal macrophages ${ }^{11}$ and this raises the possibility that the increased thromboxane synthesis in Crohn's disease may thus represent a sign of increased activation of the lipoxygenase pathways which are known to exist in human colonic mucosa. 12

Regardless of the source of the $\mathrm{TxB}_{2}$ its increased synthesis and altered relationship to prostacyclin synthesis could have several consequences which may be of pathological significance in Crohn's disease. Increased platelet aggregability and local vasoconstriction are the best characterised consequences of thromboxane synthesis although there is little prior evidence to suggest that these processes are of primary importance in Crohn's disease. Thromboxane and some lipoxygenase products may lead to reduced suppressor cell activity while other prostaglandins have an opposite effect. ${ }^{13}$ Our findings of increased $\mathrm{TxB}_{2}$ synthesis in Crohn's disease are, therefore, of particular interest in the light of a recent report of reduced suppressor cell activity in intestinal lymphocytes ${ }^{14}$ in Crohn's disease. There is an intimate relationship between macrophage prostaglandin synthesis and lymphocyte function ${ }^{15}$ and local prostaglandin synthesis is known to have potent effects upon the functional characteristics of intra-mucosal lymphocytes. ${ }^{16}$ Enhanced thromboxane synthesis by macrophages in Crohn's disease may thus play an important role in the downward modulation of intra-mucosal suppressor cell function.

Additionally prostaglandins have been proposed to have 'cytoprotective' properties in the colon ${ }^{17}$ as they do in the stomach. The evidence for prostaglandin 'cytoprotection' in the colon is at present rather limited but in the stomach there is abundant evidence that $\mathrm{PGE}_{2}$ and prostacyclin protect against ulceration and cell damage $;{ }^{18}$ conversely, excessive thromboxane synthesis is associated with necrosis and ulceration. ${ }^{19}$ The finding of increased thromboxane synthesis and an alteration in the balance of 6-keto $\mathrm{PGF}_{1 \alpha}$ and $\mathrm{TxB}_{2}$ synthesis raises the possibility of analogous consequences in the colon. Such a conclusion, however, must remain speculative as substantial doubt exists whether prostacyclin has any action affecting the colon which can reasonably be described as cytoprotection.

Prostaglandin synthesis in inflammatory bowel disease has previously been seen in terms of the 
genesis of the mucosal inflammation and a global increase in the synthesis of prostanoids. We have used the ratio of prostacyclin to thromboxane synthesis to draw attention to the importance of the balance between synthesis of prostanoids with differing inflammatory, immunological, or perhaps 'cytoprotective' properties. The effect of treatment on this balance requires further clarification. Other imbalances might have been apparent if lipoxygenase products had been measured and the results emphasise the need to define further the spectrum of potential inflammatory mediators synthesised by the colonic mucosa in health and disease.

\section{References}

1 Gould SR. Assay of prostaglandin-like substances in faeces and their measurement in ulcerative colitis. Prostaglandins 1976; 11: 489-97.

2 Sharon P, Ligumsky M, Rachmilewitz D, Zor U. Role of prostaglandins in ulcerative colitis; enhanced production during active disease and inhibition of sulfasalazine. Gastroenterology 1978; 75: 638-40.

3 Hawkey CJ. Truelove SC. Effect of prednisolone on prostaglandin synthesis by rectal mucosa in ulcerative colitis: investigation by lamina flow bioassay and radio-immunoassay. Gut 1981; 22: 190-3.

4 Ligumsky M, Karmeli F, Sharon P, Zor U, Cohen F, Rachmilewitz D. Enhanced thromboxane $\mathrm{A}_{2}$ and prostacyclin production by cultured rectal mucosa in ulcerative colitis and its inhibition by steroids and sulfasalazine. Gastroenterology 1981; 81: 444-9.

5 Moncada S, Vane JR. Pharmacology and endogenous roles of prostaglandin endoperoxides, thromboxane $A_{2}$, and prostacyclin. Pharmacol Rev 1979; 30: 293331.

6 Rachmilewitz D, Ligumsky M, Haimovitz A, Treves AJ. Prostanoid synthesis by cultured peripheral blood mononuclear cells in inflammatory diseases of the bowel. Gastroenterology 1982; 82: 673-9.

7 Smith PR, Dawson DJ, Swan CH. Prostaglandin synthetase activity in acute ulcerative colitis: effects of treatment with sulphasalazine, codeine phosphate and prednisolone. Gut 1979; 20: 8()2-5.

8 Hoult JRS, Page H. 5-amino-salicylic acid a co-factor for colonic prostaglandin synthesis? Lancet 1981; 2: 255.

9 Hoult JRS, Moore PK. Sulphasalazine is a potent inhibitor of prostaglandin 15 hydroxydehydrogenase: possible basis for therapeutic action in ulcerative colitis. Br J Pharmacol 1978; 64: 6-8.

10 Morley J, Bray A, Jones RW, Nugteren DH, Vandorp DA. Prostaglandin and thromboxane production by human and guinea pig macrophages and lymphocytes. Prostaglandins 1979; 17: 729-35.

11 Fuerstein N, Foegh M, Ramwell PW. Leukotrienes C4 and D4 induce prostaglandin and thromboxane release from rat peritoneal macrophages. Br J Pharmacol 1981; 72: 389-91.

12 Hawkey CJ, Boughton-Smith NK, Whittle BJR. Synthesis of lipoxygenase and cyclo-oxygenase products by human colonic mucosa. Gut 1982; 23: A897.

13 Kelly JP, Johnson C, Parker CW. Effect of inhibitors of arachidonic acid metabolism on mitogenesis in human lymphocytes: possible role of thromboxane and products of the lipoxygenase pathway. J Immunol 1979; 122: $1563-7$.

14 Goodacre RL, Bienenstock J. Reduced suppressor cell function in intestinal lymphocytes from patients with Crohn's disease. Gastroenterology 1982; 82: 653-8.

15 Gordon D, Bray MA, Morley J. Control of lymphokine secretion by prostaglandins. Nature 1976; 262: 401-2.

16 Bland PW, Richens ER, Britton DC, Lloyd JV. Isolation and purification of human large bowel mucosal lymphoid cells: effect of separation technique on functional characteristics. Gut 1979; 20: 1037-46.

17 Robert A, Nezamis JE, Lancaster C, Hanchar A. Prevention through cytoprotection of clindamycin induced colitis in hamsters with 16:16 dimethyl $\mathrm{PGE}_{2}$. Gastroenterology 1980; 78: 1245A.

18 Robert A. Cytoprotection by prostaglandins. Gastroenterology 1979; 77: 761-7.

19 Whittle BJR, Kauffan GL, Moncada S. Vasoconstriction with thromboxane $\mathbf{A}_{2}$ induces ulceration of the gastric mucosa. Nature 1981; 292: 472-4. 